\title{
Impact of the social deprivation on the psychosocial difficulties of pediatric cancer survivors: a prospective multicentric study
}

\author{
Fanny Delehaye ${ }^{1}$, Olivier Dejardin ${ }^{2}$, isabelle pellier ${ }^{3}$, Ludivine Launay ${ }^{4}$, Maxime Esvan $^{5}$,

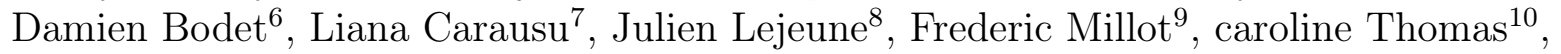 \\ Virginie Gandemer ${ }^{11}$, Arnaud Alves ${ }^{12}$, and julien Rod ${ }^{1}$ \\ ${ }^{1}$ Centre Hospitalier Universitaire de Caen \\ ${ }^{2}$ INSERM U1086 \\ ${ }^{3}$ chu angers \\ ${ }^{4}$ Centre François Baclesse Centre de Lutte Contre le Cancer \\ ${ }^{5}$ Univ Rennes, CHU Rennes, Inserm, CIC 1414 \\ ${ }^{6} \mathrm{CHU}$ de Caen \\ ${ }^{7} \mathrm{CHU}$ Brest \\ ${ }^{8} \mathrm{CHRU}$ de Tours \\ ${ }^{9} \mathrm{CHU}$ de Poitiers \\ ${ }^{10}$ CHU Nantes \\ ${ }^{11} \mathrm{CHU}$ Rennes \\ ${ }^{12} \mathrm{CHU}$ Caen
}

October 5, 2021

\begin{abstract}
Background The posttreatment period is a key part of the management of pediatric cancer care. At this period, psychosocial effects (scholarly and psychological difficulties) have been described in pediatric cancer patients and can be prognostic for the success of social reintegration. Psychosocial effects and their impact may be related to the household's socioeconomic background. The aim of this study was to estimate psychosocial difficulties during the posttreatment period based on a social deprivation score. Design This study is based on a prospective multicentric study database, and focused on the children who had received psychosocial evaluation during their follow-up after cancer treatment since 01/01/2013. We retrieved data on their learning and psychological difficulties. Socioeconomic status of the household was estimated by a social deprivation score. Results 1003 patients were analyzed. Learning difficulties at school were noted in $22 \%$ of patients. A greater social deprivation was significantly associated with learning difficulty $(\mathrm{OR}=1.09$ per unit of the deprivation score). Tumor relapse, treatment with hematopoietic stem cell transplantation, and diagnosis of a CNS tumor remained significant risk factors. In the subgroup analysis of children with CNS tumors, learning difficulties were increased and associated with greater social deprivation. By contrast, psychological difficulties were not associated with the deprivation score. Conclusion There is a link between SE status and learning difficulties in survivors of childhood cancer. Further investigations should be carried out to confirm these results for children with CNS tumors, which is the population of the greatest concern.
\end{abstract}

Impact of the social deprivation on the psychosocial difficulties of pediatric cancer survivors: a prospective multicentric study

Author names and affiliations:

Fanny DELEHAYE ${ }^{1,2}$, Olivier DEJARDIN ${ }^{1,3}$, Isabelle PELLIER ${ }^{4}$, Ludivine LAUNAY $^{1,5}$, Maxime ESVAN ${ }^{6}$, 
Damien BODET ${ }^{2}$, Liana CARAUSU ${ }^{7}$, Julien LEJEUNE ${ }^{8}$, Frédéric MILLOT ${ }^{9}$, Caroline THOMAS ${ }^{10}$, Virginie GANDEMER ${ }^{11}$, Arnaud ALVES ${ }^{1,12}$, Julien ROD ${ }^{1,13}$

1 U1086 INSERM "Anticipe", Caen, France

2 Department of Pediatric Hematology and Oncology, University Hospital of Caen, Caen, France.

3 Research department, University Hospital of Caen, Caen cedex, France.

4 Department of Pediatric Hematology and Oncology, University Hospital of Angers, France

5 Centre François Baclesse, Caen, France

6 CIC 1414 (Centre d'Investigation Clinique de Rennes), Rennes University, CHU Rennes, Inserm, F-35000 Rennes, France.

7 Department of Pediatric Hematology and Oncology, University Hospital of Brest, Brest, France

8 Department of Pediatric Hematology-Oncology, Clocheville Hospital, University Hospital of Tours, Tours, France

9 Pediatric Oncology Unit, CIC 802 INSERM, University Hospital of Poitiers, Poitiers, France

10 Department of Pediatric Hematology-Oncology, University Hospital of Nantes, Nantes, France

11 Department of Pediatric Hematology and Oncology, University Hospital of Rennes, Rennes, France

12 Service de Chirurgie Digestive, CHU Caen, 14000 Caen, France, Université de Caen-Normandie, 14000 Caen, France.

13 Department of Pediatric Surgery, Caen University Hospital, Avenue de la Côte de Nacre, 14000, Caen, France

\section{Corresponding author}

DELEHAYE Fanny, Department of Pediatric Hematology and Oncology, University Hospital of Caen, Avenue de la Côte de Nacre, 14000 Caen, France.

E-mail: [f.delehaye@gmail.com]

Phone 0033(0)2.31.06.44.88

\section{Word count}

Abstract: 243

Main text: 2856

Running title: Pediatric cancer survivors' psychosocial difficulties.

Key words: Psychosocial difficulties, contextual socioeconomic status, deprivation, cancer, children List of abbreviations

\begin{tabular}{ll}
\hline CNS & Central-nervous system \\
\hline EDI & European Deprivation Index \\
GOCE & Grand ouest pour les cancers de l'enfant \\
HSCT & Hematopoietic stem cell transplantation \\
IRIS & Ilôts regroupés pour l'information statistique \\
RECAPGO & Receuil des cancers pédiatriques du grand ouest \\
SES & Socioeconomic status \\
\hline
\end{tabular}




\section{Abstract \\ Background}

The posttreatment period is a key part of the management of pediatric cancer care. At this period, psychosocial effects (scholarly and psychological difficulties) have been described in pediatric cancer patients and can be prognostic for the success of social reintegration. Psychosocial effects and their impact may be related to the household's socioeconomic background. The aim of this study was to estimate psychosocial difficulties during the posttreatment period based on a social deprivation score.

\section{Design}

This study is based on a prospective multicentric study database, and focused on the children who had received psychosocial evaluation during their follow-up after cancer treatment since $01 / 01 / 2013$. We retrieved data on their learning and psychological difficulties. Socioeconomic status of the household was estimated by a social deprivation score.

\section{Results}

1003 patients were analyzed. Learning difficulties at school were noted in $22 \%$ of patients. A greater social deprivation was significantly associated with learning difficulty $(\mathrm{OR}=1.09$ per unit of the deprivation score). Tumor relapse, treatment with hematopoietic stem cell transplantation, and diagnosis of a CNS tumor remained significant risk factors. In the subgroup analysis of children with CNS tumors, learning difficulties were increased and associated with greater social deprivation. By contrast, psychological difficulties were not associated with the deprivation score.

\section{Conclusion}

There is a link between SE status and learning difficulties in survivors of childhood cancer. Further investigations should be carried out to confirm these results for children with CNS tumors, which is the population of the greatest concern.

\section{Background}

The international incidence of pediatric cancer is reported to be 140.6 cases per million children aged 0-14 years per year. In children aged 15-19 years, the incidence increases to 185.3 cases per million person-year(1). In France, these rates are reported to be 156.6 and 231.9 cases per million person-years, respectively $(2,3)$. Over time, a significant improvement in long-term survival rates of pediatric cancers has been achieved, with a current 5-year survival rate at 75\% in Europe(4). Therefore, the post-treatment period is a key part of the management, and the goal is to help the patient return to a normal life. Childhood cancer and its treatments may induce sequalae. Among them, the psychosocial affections, i.e. difficulties on educational achievement, psychological well-being, and the household's economic status are well-known and can be prognosis for the success of the patients' postcancer reintegration(5-7). Learning and psychological consequences are mostly described for children with central-nervous system (CNS) tumors, with cranial radiotherapy and with a younger age at diagnosis(5,8-12). Late repercussions are described, with survivors being at greater risk of unemployment and the later development mental disorders than their siblings(11,13). Some of these consequences may be related to the household's socioeconomic background. Socioeconomic status (SES) can influence the probability of psychosocial difficulty in addition to the ability of the family to be able to deal with its occurrence. For cancer survivors, some studies established associations among learning difficulties at school, psychological well-being and household SES(14-17). However, these studies show links that are not based on reproductible socioeconomic deprivation scores, and mainly focus on the evaluation of only one type of social difficulties and when the social reintegration is effective $(8,10,11,15,16,18-21)$. The aim of this study was to estimate the psychosocial difficulties in childhood cancer survivors at the posttreatment period based on a social deprivation score. We evaluated educational and psychological difficulties immediately after the hospital care period, a time when interventions could be possible to avoid the long-term consequences and improve social reintegration rates. 


\section{Methods}

Population

This French multicentric study focused on the children from the RECAPGO database (REcueil des CAncers Pédiatriques du Grand Ouest ) who received psychosocial evaluation. This evaluation consisted of a questionnaire that reported the learning and psychological difficulties of children. All the children included in the database were eligible to this evaluation. There was no difference noted between children who received the evaluation and those who did not according to the SES (Table 1). This questionnaire was completed by oncologist pediatricians during the years of close follow-up required after the end of the child's hospital treatment. Each difficulty was reported independently (i.e., school and psychological). We defined the psychosocial follow-up as completing at least one assessment for these two questionnaire items at least once during the follow-up period.

The RECAPGO database results from a collaboration among seven French care centers, reported as the French children's oncology study Group GOCE (Grand Ouest pour les Cancers de l'Enfant). This prospective database has been open since 01/01/2013, and aims to include all patients aged under 25 years diagnosed with a cancer, a hematological malignancy, an aplastic anemia, or a Langerhans cell histiocysis at the participating centers. We included patients recorded at diagnosis with a single tumor experience. Patients with no known address at diagnosis, those living outside of the GOCE departments, those with no estimated social deprivation score, those aged over 18 years and those with missing tumor type data were excluded. One center did not complete the psychosocial questionnaire and was excluded from the analysis (Figure 1).

Variables

We defined three types of variables (i) At each consultation during the follow-up, oncologist pediatricians carried out a psychosocial evaluation. During this specific dedicated time, pediatricians, children and their parents discuss the potential psychosocial difficulties. At the end of the discussion, a synthesis was reported using as a binary form (yes/no) to indicate if learning or psychological difficulty was present or not. Whether the patient received assistance for each difficulty was also reported. (ii) The clinical variables were composed of the patients and their tumors characteristics. We merged the tumor types into five categories; i.e., blood disorders, CNS tumors, solid tumors, bone tumors, and other tumors (iii) Two variables evaluated the SES of the children and their household. First, the European Deprivation Index (EDI) score assessed the socioeconomic environment of each patient. This deprivation indicator is constructed by selecting fundamental needs associated both with objective and subjective poverty(22). This score is determined by an ecological measure using the IRIS-scale (Ilôts Regroupés pour l'Information Statistique ), which represents the smallest French geographical area for which there is a statistical evaluation to estimate social deprivation. Based on their addresses at diagnosis, each patient can be associated with an IRIS, and thus, their EDI score can be established $(22,23)$. For interpretation, the highest EDI score is associated with greater social deprivation. Second, we used the travel time defined as the shortest time to travel by car from the patient's address to the reference care center to assess geographical disparities. A geographical information system (ArcGIS 10.5®Esri France) associated with a road map database (Navstreets@, provided by HERE and Esri France) was used for this estimation. For the RECAPGO database, each patient's EDI score and travel time to reference care center were estimated from the address at diagnosis.

\section{Statistical analysis}

Baseline characteristics of the population were described as the mean $(+/-$ standard deviation $)$ for quantitative variables and numbers (percentages) for qualitative variables. Quantitative and qualitative variables were analyzed by t-tests and chi2 tests, respectively. Two types of analysis were carried out (i) A multivariate analysis for factors associated with the probability of declaring a psychosocial difficulty at a consultation was conducted, considering mixed effects for longitudinal data. We defined at the second level the children. We defined at first level each consultation during which the psychosocial questionnaire was completed. Factors associated with a p-value $<0.10$ in the univariate analysis were considered in the multivariate logistic model. In this model, time was included as an independent variable. Additionally, subgroup analyses for children 
with blood disorders and CNS tumors were carried out (ii) We evaluated the probability of psychosocial difficulty over time by using Kaplan-Meier failure function curves. We completed a parametric survival regression model based on the same longitudinal data structure with two levels. The original date was the first consultation when the psychosocial follow-up began. However, we did not have the same follow-up duration and measurements' time between patients according to their oncologic disease. Considering this, time to consultation was considered as an independent factor to assess the potential influence of the delay when the measurement is taking place.

All statistical analyses were performed with STATA software V14 and a p-value $<0.05$ was considered to denote statistical significance.

We defined the primary endpoint as the probability of presenting at a consultation with a learning difficulty at school. The secondary endpoint was to evaluate the probability of presenting a learning difficulty over time. Other psychosocial difficulties were also analyzed for further explorations.

This study was based on a database supported by the Commission Nationale de l'Informatique et des Libertés (CNIL) registered under the $\mathrm{N}^{\circ} 912302$.

\section{Results}

Overall, 1618 patients were included from six centers, of which 1003 (62\%) received a psychosocial followup. Learning and psychological were evaluated in 2971 and 3320 consultations, respectively. Academic and psychological supports was evaluated in 2625 and 3320 consultations, respectively (Figure 1). The population who received a psychosocial follow-up did not differ substantially from the entire population of the study (Table 1). However, the children who received a psychosocial follow-up were less frequently treated with radiotherapy. The rate of children followed differed significantly between the centers, from $30 \%$ to $80 \%$. This rate differed according to vital status and relapse.

Learning difficulties at school

Learning difficulties at school were self-reported by 224 children (22\%) (Table 2). Greater social deprivation was significantly associated with a learning difficulty at school; however, the increased geographic distance evaluated by the total travel time was not. Additionally, tumor relapse, treatment with hematopoietic stem cell transplantation (HSCT), and the diagnosis of a CNS tumor remained significant risk factors. A significant interaction was found between the centers and the tumor type, notably for CNS tumors $(\mathrm{p}<0.001)$. Threehundred seventy-one children received a specific assistance with academic support, of which $74 \%$ reported difficulties at school. Academic support was mainly provided for children with CNS tumors $(\mathrm{p}<0.001)$. Children with CNS tumors benefited from $52 \%$ of the academic supports retrieved for the entire population. Although learning difficulties were significantly associated with greater social deprivation, the probability of benefitting from a support was not. However, there was an important correlation between the learning disabilities and academic support $\left(\mathrm{R}^{2}=0.79\right)$.

Over time, two factors were significantly associated with the probability of having a learning difficulty at school. First, CNS tumors were a significant risk factor in comparison with other types of tumors, with a hazard ratio of 4.48 (CI: $2.79-7.21$ ) (Figure 2A). Second, the tumor relapse was a significant risk factor, with a hazard ratio of 1.80 (CI: $1.05-3.09$ ) (Figure 2B).

Ninety-two children with a CNS tumor reported a learning disability at school (46\%). The risk factors that were significantly associated were greater social deprivation, tumor relapse, treatment by chemotherapy, and an increased delay in the consultation. In contrast, the surgical management was protective (Table 3). Neither the type of tumor nor its localization had a significant impact on the risk of having a learning difficulty. The probability of benefiting from academic support was not influenced by the EDI score in this population. In children who declared learning disabilities, $19 \%$ of them did not receive support. Over time, only treatment by chemotherapy remained a risk factor for learning difficulty at school, with a hazard ratio of 2.54 (CI: 1.52 $-4.23)$. 
Seventy-eight children with blood disorders reported a learning difficulty at school (17\%). The risk factors were tumor relapse and management by HSCT (Supplemental appendix 1). An increased delay in the consultation and female sex were protective factors. Female sex remained the only significant and protective factor against developing a learning disability over time $(\mathrm{HR}=0.37, \mathrm{CI}: 0.19-0.73)$. However, a trend was observed with tumor relapse status $(\mathrm{p}=0.09)$.

Psychological difficulties

Greater social deprivation was not significantly associated with the report of psychological difficulties at consultation. However, significant differences were noted between care centers. Psychological difficulties were reported in 196 children (19.5\%) (Supplemental appendix 2). At consultation, associated risk factors were tumor relapse and the diagnosis of bone tumors. Psychological support was reported for $70 \%$ of the patients. Over time, the diagnosis of a bone tumor was a risk factor for psychological difficulty (HR = 2.04, CI: 1.05 $-3.96)$, whereas a solid tumor was a protective factor $(\mathrm{HR}=0.53, \mathrm{CI}: 0.30-0.93)$. Nethertheless, a trend could be noted for the increased EDI-score $(\mathrm{p}=0.064)$.

\section{Discussion}

The results of this multicentric study show that greater social deprivation and therefore poverty, is associated with learning difficulties in survivors after cancer treatment.

Learning difficulties were reported in $22 \%$ of the children in our study, but higher rates have been noted in the literature $(21,24)$. Survivors are at greater risk for presenting learning difficulties with scholarly consequences $(10,15,21,24)$, ultimately leading to a lower overall quality of life(25). School absenteeism induced by cancer care is the most commonly reported productivity $\operatorname{loss}(8,26)$. Even if we did not evaluate the rate of absenteeism in our cohort, we found that tumor relapse was a risk factor. The tumor relapse frequently requires extended hospital stays and therefore, induces school absenteeism.

In our study, the probability of declaring a learning difficulty at school was associated with greater social deprivation. Irrespective of the disease, there is a strong relationship between SES and scholarly achievement(27-29). This supports our results and those reported in the literature, even though different methods were used to estimate the SES across studies $(14,15,25)$. Parental involvement should also be considered as a factor influencing academic achievement(30). Children with CNS tumors are the population of greatest concern. CNS tumors and CNS directed therapies (i.e., cranial radiotherapy and intrathecal chemotherapy) increase the risk of adverse psychosocial consequences $(7,24,31)$. Academic and neurocognitive difficulties are described, as well as higher rates of school absenteeism $(8,9,21)$. We did not confirm the inherent risk of radiotherapy in our CNS subgroup analysis, probably due to the lack of children receiving irradiation followed in our study. In this CNS subgroup, social deprivation remained a prognostic factor for learning difficulties, an observation also shown by Ach et al.(32).

HSCT was a risk factor for learning disabilities, in agreement with previous reports that a high number of children receiving HSCT present with academic difficulties and do not graduate high school(33).

Learning difficulties may be induced by a lack integration into the school environment, as adequate socialization is a contributing factor to academic success(29). Our data collection precludes this analysis; however, Duan et al. demonstrated that greater social deprivation negatively influences the relationship between academic socialization and academic achievement(29). Survivors suffer from poor social integration, potentiated by absenteeism $(21,24,26,34)$. These difficulties are described more frequently in patients with CNS tumors.

Due to the high risk for learning disabilities in survivors, school-organized educational support remains necessary. Thirty-six percent of children received a such support in our study, a rate slightly higher than that reported in the literature, which ranged from $20 \%$ to $32.5 \%(24,31,35)$. Although greater deprivation was associated with learning disabilities, it was not associated with the probability of benefiting from academic support. Academic support seems to be equally shared between children according to the EDI score, whereas we showed a higher need in deprived areas. 
Our analysis of psychological difficulties did not reveal an association with social deprivation. However, SES may participate in causal mechanisms for psychological problems $(16,19)$. One study revealed that neighborhood SES affected the probability of benefiting from psychological support(36). Children with CNS tumors are at greater risk for psychological effects, whereas our results indicated that children with bone tumors were at risk(11,19). The psychological effects of bone cancer can be explained by physical damage secondary to the surgery, inducing vulnerability related to physical performance limitations, disruption to routine activities, and diminished ability to attend work or school $(37,38)$.

This study is based on a prospective cohort and allowed us to evaluate the overall psychosocial status of survivors after hospital treatment and the evolution of psychosocial effects over time. However, this study presents some limitations.

Our population was based on a database which aims to represent all the children treated for cancer in the GOCE departments. However, one center was excluded from the study because it did not participate to patients' psychosocial evaluation. This excluded $6 \%$ of the children of the cohort and therefore, we cannot exclude a selection bias (Figure 1).

Evaluation biases were present as a result of the variables' measurement. Each outcome was evaluated during a consultation with the oncologist pediatrician at a time when the clinical evaluation remained the main objective to ensure remission persistence. The outcomes were self-reported by the parents and children, and the subjective aspect of self-reports cannot be ruled out. In addition, the data was only collected at the posttreatment period and no baseline at diagnosis was available. This precludes any analysis to evaluate the evolution of psychosocial difficulties before and after the cancer management. This limits us to an observation of post-treatment difficulties without taking account of some potential predisposed conditions. Indeed, in children with CNS tumors, preexisting predisposition syndromes (e.g., type one fibromatosis) can induce learning and psychological effects, as well as the tumor symptoms themselves (39-41). However, the evaluation of psychosocial status at diagnosis may be difficult. This time is a period when clinical management remains the absolute priority. This can explain the choice of investigators to defer the psychosocial evaluation to after treatment.

The measurement of social deprivation using the EDI score assesses the SES of the environment of each patient and can induce an ecological bias. This measurement considers homogeneity between children living in the same IRIS and could induce misclassification and underestimate the effect of SES. Data on individual deprivation could be a complementary method to precise the SES of children, but were not available in the database.

Our statistical analysis is another limitation. Each child had a different follow-up duration, which limited our utilization of the Cox model. These different follow-up times resulted in missing data organized in a monotone structure, resulting from dropout, and limited the use of multiple imputations to consider the missing data. Additionally, multiple imputation is not recommended for longitudinal data analysis because of the lack of obvious benefit in this context(42).

\section{Conclusion}

This study establishes some basis and suggests further investigations. Children with CNS tumors are the population most at risk for psychosocial difficulties and social deprivation seems to be significantly associated with these effects $(7,8,11,19,21,24,31,35)$. A focus on this group could be interesting to detail the content of the difficulties of these patients, and confirm the impact of the SES and complete this evaluation with individual socioeconomic indicators. This may help to confirm and precise some of the problems noted by various patients. Therefore, we could offer solutions to reduce these inequalities.

\section{Disclosure of interest}

Authors have nothing to disclose.

\section{Funding}


No specific funding sources were obtained for this work. The French RECAPGO cancer database is supported by a grant from SFCE (Société Française des Cancers de l'Enfant).

\section{Acknowledgments}

Authors gratefully acknowledge patients and their families. Additionally, the authors acknowledge Dr Bouvier Véronique (U1086 INSERM UCBN, Caen, France) for her valued help and advices regarding this paper. We gratefully acknowledge the AJE editing services for help in manuscript edition.

\section{References}

1. Steliarova-Foucher E, Colombet M, Ries LAG, Moreno F, Dolya A, Bray F, et al. International incidence of childhood cancer, 2001-10: a population-based registry study. The Lancet Oncology. juin 2017;18(6):719-31.

2. Lacour B, Guyot-Goubin A, Guissou S, Bellec S, Desandes E, Clavel J. Incidence of childhood cancer in France: National Children Cancer Registries, 2000-2004. European Journal of Cancer Prevention. mai 2010;19(3):173-81.

3. Raze T, Lacour B, Cowppli-Bony A, Delafosse P, Velten M, Tretarre B, et al. Cancer Among Adolescents and Young Adults Between 2000 and 2016 in France: Incidence and Improved Survival. Journal of Adolescent and Young Adult Oncology. 1 fevr 2021;10(1):29-45.

4. Gatta G, Capocaccia R, Stiller C, Kaatsch P, Berrino F, Terenziani M, et al. Childhood Cancer Survival Trends in Europe: A EUROCARE Working Group Study. JCO. 1 juin 2005;23(16):3742-51.

5. Frederiksen LE, Mader L, Feychting M, Mogensen H, Madanat-Harjuoja L, Malila N, et al. Surviving childhood cancer: a systematic review of studies on risk and determinants of adverse socioeconomic outcomes. Int J Cancer. 15 avr 2019;144(8):1796-823.

6. Limburg H, Shaw AK, McBride ML. Impact of childhood cancer on parental employment and sources of income: A Canadian pilot study: Childhood Cancer and Parental Employment and Income. Pediatr Blood Cancer. juill 2008;51(1):93-8.

7. Brinkman TM, Recklitis CJ, Michel G, Grootenhuis MA, Klosky JL. Psychological Symptoms, Social Outcomes, Socioeconomic Attainment, and Health Behaviors Among Survivors of Childhood Cancer: Current State of the Literature. JCO. 20 juill 2018;36(21):2190-7.

8. French AE, Tsangaris E, Barrera M, Guger S, Brown R, Urbach S, et al. School Attendance in Childhood Cancer Survivors and Their Siblings. The Journal of Pediatrics. janv 2013;162(1):160-5.

9. Bonneau J, Dugas K, Louis A, Morel L, Toughza J, Frappaz D. Scolarite et devenir social apres un cancer dans l'enfance. Bulletin du Cancer. juill 2015;102(7-8):691-7.

10. Andersen KK, Duun-Henriksen AK, Frederiksen MH, Winther JF. Ninth grade school performance in Danish childhood cancer survivors. Br J Cancer. janv 2017;116(3):398-404.

11. Lund LW, Winther JF, Dalton SO, Cederkvist L, Jeppesen P, Deltour I, et al. Hospital contact for mental disorders in survivors of childhood cancer and their siblings in Denmark: a population-based cohort study. The Lancet Oncology. sept 2013;14(10):971-80.

12. Molcho M, D'Eath M, Alforque Thomas A, Sharp L. Educational attainment of childhood cancer survivors: A systematic review. Cancer Med. juin 2019;8(6):3182-95.

13. Mader L, Michel G, Roser K. Unemployment Following Childhood Cancer. Deutsches Aerzteblatt Online [Internet]. 24 nov 2017 [cite 26 mars 2021]; Disponible sur: https://www.aerzteblatt.de/10.3238/arztebl.2017.0805

14. Patel SK, Johansen C, Gold AO, Delgado N, Xu S, Dennis J. Social-ecological predictors of school functioning in Hispanic children treated for cancer with central nervous system-directed ther- 
apies. Pediatr Blood Cancer [Internet]. oct 2020 [cite 26 janv 2021];67(10). Disponible sur: https://onlinelibrary.wiley.com/doi/10.1002/pbc.28320

15. Bonneau J, Lebreton J, Taque S, Chappe C, Bayart S, Edan C, et al. School Performance of Childhood Cancer Survivors: Mind the Teenagers! The Journal of Pediatrics. janv 2011;158(1):135-41.

16. Zebrack BJ, Zevon MA, Turk N, Nagarajan R, Whitton J, Robison LL, et al. Psychological distress in long-term survivors of solid tumors diagnosed in childhood: A report from the childhood cancer survivor study. Pediatr Blood Cancer. juill 2007;49(1):47-51.

17. Zebrack BJ, Zeltzer LK, Whitton J, Mertens AC, Odom L, Berkow R, et al. Psychological Outcomes in Long-Term Survivors of Childhood Leukemia, Hodgkin's Disease, and Non-Hodgkin's Lymphoma: A Report From the Childhood Cancer Survivor Study. PEDIATRICS. 1 juill 2002;110(1):42-52.

18. de Boer AGEM, Verbeek JHAM, van Dijk FJH. Adult survivors of childhood cancer and unemployment: A metaanalysis. Cancer. 1 juill 2006;107(1):1-11.

19. Lund LW, Schmiegelow K, Rechnitzer C, Johansen C. A systematic review of studies on psychosocial late effects of childhood cancer: Structures of society and methodological pitfalls may challenge the conclusions: Psychosocial Effects of Childhood Cancer. Pediatr Blood Cancer. avr 2011;56(4):532-43.

20. Gummersall T, Skaczkowski G, Wilson C. Childhood cancer, age at diagnosis and educational attainment: A meta-analysis. Critical Reviews in Oncology/Hematology. janv 2020;145:102838.

21. Park M, Park HJ, Lee JM, Ju HY, Park BK, Yu E-S, et al. School performance of childhood cancer survivors in Korea: A multi-institutional study on behalf of the Korean Society of Pediatric Hematology and Oncology. Psycho-Oncology. sept 2018;27(9):2257-64.

22. Pornet C, Delpierre C, Dejardin O, Grosclaude P, Launay L, Guittet L, et al. Construction of an adaptable European transnational ecological deprivation index: the French version. J Epidemiol Community Health. nov 2012;66(11):982-9.

23. Guillaume E, Pornet C, Dejardin O, Launay L, Lillini R, Vercelli M, et al. Development of a cross-cultural deprivation index in five European countries. J Epidemiol Community Health. mai 2016;70(5):493-9.

24. Barrera M, Shaw AK, Speechley KN, Maunsell E, Pogany L. Educational and social late effects of childhood cancer and related clinical, personal, and familial characteristics. Cancer. 15 oct 2005;104(8):175160 .

25. Litzelman K, Barker E, Catrine K, Puccetti D, Possin P, Witt WP. Socioeconomic disparities in the quality of life in children with cancer or brain tumors: the mediating role of family factors: SES, family functioning, and QOL among children with cancer. Psycho-Oncology. mai 2013;22(5):1081-8.

26. Tsimicalis A, Genest L, Stevens B, Ungar WJ, Barr R. The Impact of a Childhood Cancer Diagnosis on the Children and Siblings' School Attendance, Performance, and Activities: A Qualitative Descriptive Study. J Pediatr Oncol Nurs. mars 2018;35(2):118-31.

27. Battle J, Lewis M. The Increasing Significance of Class: The Relative Effects of Race and Socioeconomic Status on Academic Achievement. Journal of Poverty. mars 2002;6(2):21-35.

28. Sirin SR. Socioeconomic Status and Academic Achievement: A Meta-Analytic Review of Research. Review of Educational Research. sept 2005;75(3):417-53.

29. Duan W, Guan Y, Bu H. The Effect of Parental Involvement and Socioeconomic Status on Junior School Students' Academic Achievement and School Behavior in China. Front Psychol. 19 juin 2018;9:952.

30. Fan X, Chen M. Parental Involvement and Students' Academic Achievement: A Meta-Analysis. Educational Psychology Review. 2001;13(1):1-22. 
31. Mitby PA, Robison LL, Whitton JA, Zevon MA, Gibbs IC, Tersak JM, et al. Utilization of special education services and educational attainment among long-term survivors of childhood cancer: A report from the childhood cancer survivor study. Cancer. 15 fevr 2003;97(4):1115-26.

32. Ach E, Gerhardt CA, Barrera M, Kupst MJ, Meyer EA, Patenaude AF, et al. Family factors associated with academic achievement deficits in pediatric brain tumor survivors: Academic achievement in survivors. Psycho-Oncology. aout 2013;22(8):1731-7.

33. Ness KK, Bhatia S, Baker KS, Francisco L, Carter A, Forman SJ, et al. Performance Limitations and Participation Restrictions Among Childhood Cancer Survivors Treated With Hematopoietic Stem Cell Transplantation: The Bone Marrow Transplant Survivor Study. Arch Pediatr Adolesc Med. 1 aout 2005;159(8):706.

34. McLoone JK, Wakefield CE, Cohn RJ. Childhood cancer survivors' school (re)entry: Australian parents' perceptions: School (re)entry after childhood cancer. Eur J Cancer Care (Engl). juill 2013;22(4):484-92.

35. Lorenzi M, McMillan AJ, Siegel LS, Zumbo BD, Glickman V, Spinelli JJ, et al. Educational outcomes among survivors of childhood cancer in British Columbia, Canada: Report of the Childhood/Adolescent/Young Adult Cancer Survivors (CAYACS) Program. Cancer. 15 mai 2009;115(10):2234-45.

36. Lau N, Bradford MC, Steineck A, Scott S, Bona K, Yi-Frazier JP, et al. Examining key sociodemographic characteristics of adolescents and young adults with cancer: A post hoc analysis of the Promoting Resilience in Stress Management randomized clinical trial. Palliat Med. mars 2020;34(3):336-48.

37. Ness KK, Gurney JG. Adverse Late Effects of Childhood Cancer and Its Treatment on Health and Performance. Annu Rev Public Health. avr 2007;28(1):279-302.

38. Ness KK, Mertens AC, Hudson MM, Wall MM, Leisenring WM, Oeffinger KC, et al. Limitations on Physical Performance and Daily Activities among Long-Term Survivors of Childhood Cancer. Ann Intern Med. 1 nov 2005;143(9):639.

39. Zumel-Marne A, Kundi M, Castano-Vinyals G, Alguacil J, Petridou ET, Georgakis MK, et al. Clinical presentation of young people (10-24 years old) with brain tumors: results from the international MOBI-Kids study. J Neurooncol. avr 2020;147(2):427-40.

40. North K, Joy P, Yuille D, Cocks N, Mobbs E, Hutchins P, et al. Specific learning disability in children with neurofibromatosis type 1: Significance of MRI abnormalities. Neurology. 1 mai 1994;44(5):878-878.

41. Varan A, Şen H, Aydın B, Yalçın B, Kutluk T, Akyüz C. Neurofibromatosis type 1 and malignancy in childhood: Neurofibromatosis type 1 and malignancy in childhood. Clin Genet. mars 2016;89(3):341-5.

42. Twisk J, de Boer M, de Vente W, Heymans M. Multiple imputation of missing values was not necessary before performing a longitudinal mixed-model analysis. Journal of Clinical Epidemiology. sept 2013;66(9):1022-8.

\section{Legends}

FIGURE 1 Flow chart of the study

\section{FIGURE 2}

A Probability of acquiring a learning disability at school over time according to tumor type

B Probability of acquiring a learning disability at school over time according to tumor relapse 


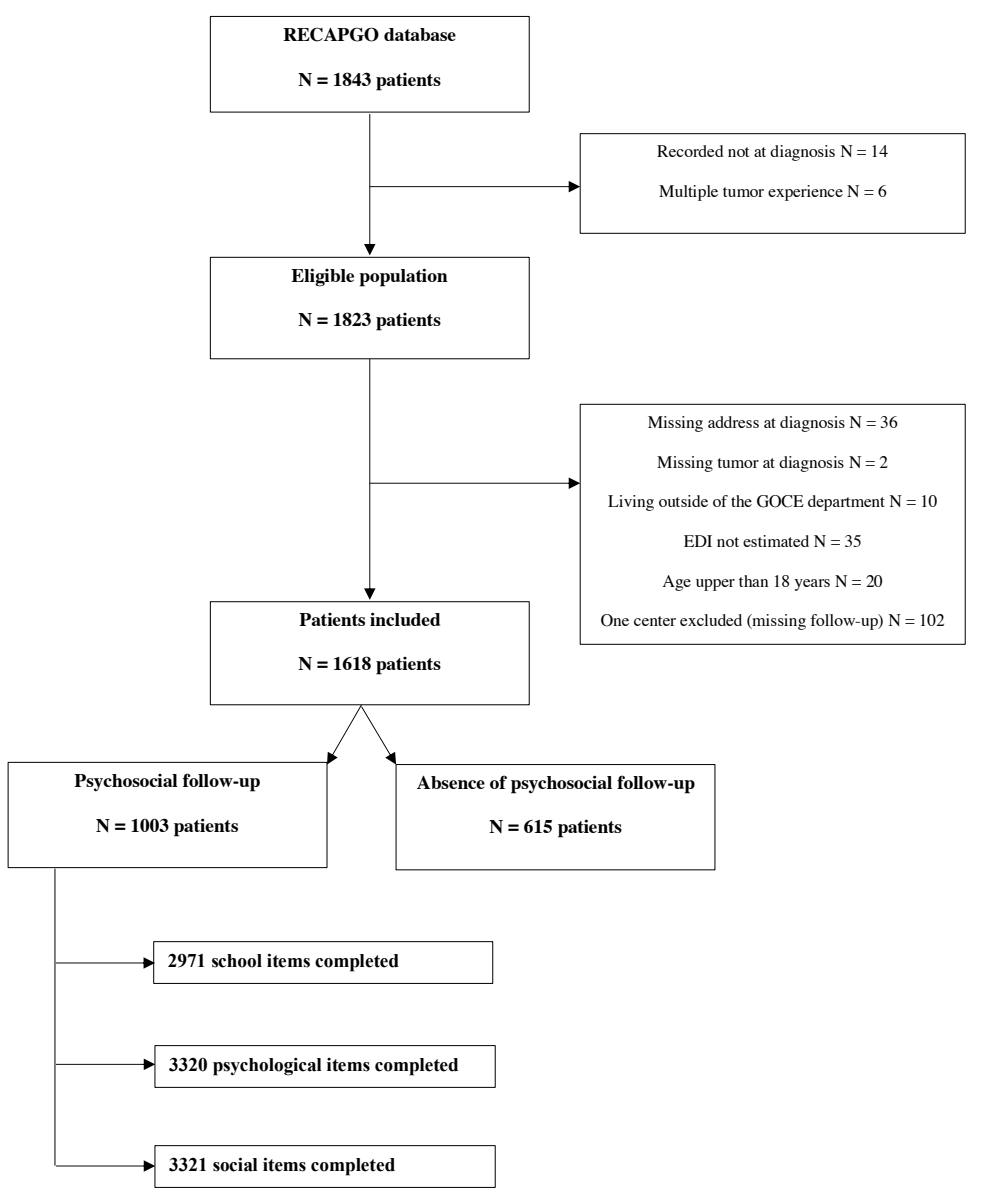


A

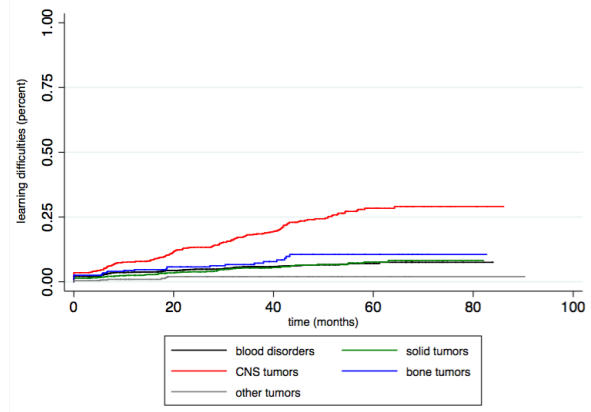

B

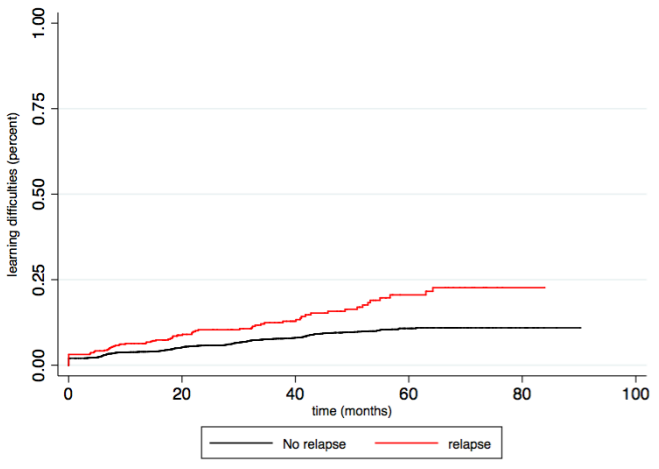

